\title{
Incorrect application of the epicycloid equation to the planetary mechanism of the cotton harvester
}

\author{
Khabibulla Turanov ${ }^{1, *}$, and Mukhaya Shaumarova ${ }^{2}$ \\ ${ }^{1}$ Tashkent State Technical University named after Islam Karimov, University Str., 2, 100174, \\ Tashkent. Uzbekistan \\ ${ }^{2}$ Tashkent Institute of Irrigation and Agricultural Mechanization Engineers, Qori Niyoziy Str., 39, \\ 100000, Tashkent, Uzbekistan
}

\begin{abstract}
The content of the paper is based on the mathematical construction of the parametric equation of the epi- and hypocycloid curve described by a circle point. The purpose of the paper is to present the equations of epi- and hypocycloids in a parametric form in relation to the epi- and hypocyclic mechanism in a form convenient for calculation; to present the results of computational experiments on constructing phase trajectories of motion of a moving point of an epi- and hypocycloid. A detailed analysis of the analytical model of epi- and hypocycloids circumscribed by a point of a circle (on a moving circle) has been made. The equations of epi- and hypocycloids are presented in parametric form as applied to the epi- and hypocyclic mechanism in a form convenient for calculation. The results of studies on the construction of phase trajectories of a moving point of an epi- and hypocycloid with an analysis of the obtained curves are presented. The analytical model of epi- and hypocycloids is of practical importance, since it allows designing geared linkage mechanisms formed by attaching two-wire Assur group of various modifications to the planetary mechanism, as the primary mechanism.
\end{abstract}

\section{Introduction}

The geared linkage mechanisms formed by fastening tightly to the connecting rod two-wire Assur groups of the second type (RRT: rotational - rotational - translational) of the satellite of the differential mechanism are well known (see Fig. 179 in [1] and, which is one and the same, in task 16.36 [2]). An analysis of works [3-16] showed that the geared linkage mechanism (according to N.E. Zhukovsky, "as a crank") pivotally connected to the satellite of the planetary mechanism of the two-wire Assur group of the second kind is not known at all, except in [14] (fig. 1).

\footnotetext{
* Corresponding author: khturanov@yandex.ru
} 


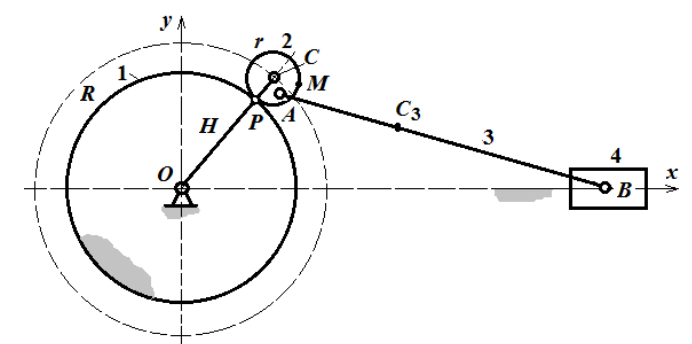

1 - fixed gear wheel; 2 - a gear wheel with a movable axis (satellite); $\mathrm{H}$ - pinion cage; 3 - connecting rod; 4 slider.

Fig. 1. Scheme of the geared linkage mechanism.

Notations in fig. 1: $O$ - the origin; $P$ - the contact point of the satellite 2 with the fixed wheel $1 ; C$ - the axis of rotation of the gear wheel $2 ; A$ - hinge in the satellite 2 for the movable connection of the connecting rod 3; $R$ and $r$ - the radii of the gear wheels 1 and 2; $A B$ - connecting rod; $C_{3}$ - the center of gravity of the connecting rod.

It is well known in mathematics (see p. 108 in [17], p. 242 in [18], p. 1010 in [19], [20]) that the epicycloid equation in parametric form is:

$$
\left.\begin{array}{l}
x_{M}=(A+a) \cos \varphi-a \cos \left[\frac{(A+a) \cdot \varphi}{a}\right] ; \\
y_{M}=(A+a) \sin \varphi-a \sin \left[\frac{(A+a) \cdot \varphi}{a}\right]
\end{array}\right\}
$$

Here $x_{M}$ and $y_{M}$ are the coordinates of the point $M$ lying on the moving circle; $A$ and $a$ the radii of a fixed and moving circle, respectively; $\varphi$ - the angle $\angle C O x$ by which the center of the moving circle is rotated (see Fig. 123 in [18]),).

The shape of the epicycloid curve depends on the ratio $\frac{A}{a}=m$, i.e. $m$ - the number of branches that "go around" a fixed circle outside it. If $m$ is an integer, then the curve consists of $m$ branches that "go around" the fixed circle.

In relation to epicyclic (or planetary) mechanisms, equation (1) in parametric form can be represented as:

$$
\left.\begin{array}{l}
x_{M}=(R+r) \cos \varphi_{H}-r \cos \left[\frac{(R+r) \cdot \varphi_{H}}{r}\right] ; \\
y_{M}=(R+r) \sin \varphi_{H}-r \sin \left[\frac{(R+r) \cdot \varphi_{H}}{r}\right]
\end{array}\right\}
$$

Here $R$ and $r$ are the radii of a fixed and a moving circle, respectively; $\varphi_{H}$ - the angle $\angle C O x$ by which the center of the moving circle is rotated.

Varieties of equation (2) are presented in [19, 20].

Planetary mechanisms are widely used in drives of vertically spindle drums of cotton harvesters (see p. 162 in [16], p. 14 in [21]). However, the practical application of the epicycloid equation in parametric form in the form (1) and/or (2) in literature $[1-11,15,16]$ is absent, except in [14, 22]. For example, in [22], an attempt was made to apply equation (2) in the planetary mechanisms of cotton harvesters, and in [14] - in six-link mechanisms formed on the basis of the planetary gear.

At the same time, as applied to the drives of vertically spindle drums of cotton harvesters as planetary mechanisms, equation $(2)$ in $[12,20]$ is presented in the following 
form (see, for example, equation (5.1) in [14] and (8.77) in 22]):

$$
\left.\begin{array}{l}
x_{M}=R_{H} \cos \varphi_{H}-R_{2} \cos \left[\left(1+i_{21}\right) \cdot \varphi_{H}\right] \\
y_{M}=R_{H} \sin \varphi_{H}-R_{2} \sin \left[\left(1+i_{21}\right) \cdot \varphi_{H}\right] .
\end{array}\right\}
$$

Here $R_{H}=R_{1}+R_{2}$ - length the pinion cage $H$ (here, $R_{l}$ and $R_{2}$ correspond to $R$ and $r$ in equation (2)); $i_{21}$ - the gear ratio determined through the radii of the fixed and moving circles, taking into account that the gearing is external (i.e. taking into account the sign of $i_{21}$ in the form $\left.i_{21}=-\frac{R_{1}}{R_{2}}\right)$ and/or internal, although the gear wheel 1 is fixed $\left(\omega_{1}=0\right)$.

Comparing equations (2) and (3), we note that an error was made in [14, 22], which consists in the impossibility of replacing the number of branches $m=R / r$ that "go around" the fixed circle outside it by the gear ratio $i_{21}$ taking into account its negative sign and the fact that $i_{21}$ through the relationship of angular velocities does not make sense, since $i_{21}=\frac{\omega_{1}}{\omega_{2}}=0$.

In addition, the epicycloid equations in parametric form (3) with additions to the arguments of it subtracted $i_{21} \varphi_{1}$ with respect to the differential mechanism are written in the form (see equations (8.68) in [22]):

$$
\left.\begin{array}{l}
x_{M}=\left(R_{1}+R_{2}\right) \cos \varphi_{H}-R_{2} \cos \left[\left(1+i_{21}\right) \cdot \varphi_{H}+i_{21} \cdot \varphi_{1}\right] ; \\
y_{M}=\left(R_{1}+R_{2}\right) \sin \varphi_{H}-R_{2} \sin \left[\left(1+i_{21}\right) \cdot \varphi_{H}+i_{21} \cdot \varphi_{1}\right] .
\end{array}\right\}
$$

Here $\varphi_{1}$ - angle of rotation of the moving circle (gear wheel 1) (see Fig. 8.3 on page 273 in [22]).

It is obvious that any scientifically unjustified correction in the form of a replacement of the number of branches $m$ of the epicycloid by the gear ratio $i_{21}$, taking into account its negative sign, introduced into the epicycloid equations in parametric form (see equation (1) or (2)) will affect all subsequent calculations. For example, due to the incorrectness of the mathematical notation of equation (3) in [14, 22], all subsequent calculations for determining the speed and acceleration of the moving point $M$ of the satellite (see, for example, equations (5.5) and (5.6) in [14] and (8.75), (8.77) in [22]) are scientifically inconsistent.

Thus, the evidence of the inadmissibility of applying an equation of type (3) to planetary mechanisms $[14,22]$ and (4) to differential mechanisms [20] is revealed, which confirms the relevance of studies on the use of the epi- and hypocycloid equation in parametric form in the mechanisms of various technological purposes.

\section{Object}

Present the equations of epi- and hypocycloids in parametric form in relation to the epi- and hypocyclic mechanism in a form convenient for calculation.

Present the results of computational experiments on constructing phase trajectories of motion of a moving point $M$ of the epi- and hypocycloid in the Mathcad system. 


\section{Method of research}

The research methods are based on the preparation of a parametric equation (in the form of transcendental functions) of the curve (epi- and hypocycloids) circumscribed by a point of a circle, assuming that this circle should roll without sliding along another circle (for example, a fixed circle) [17-20].

\section{Research results}

\subsection{Analysis of analytical models of epi- and hypocycloids}

Below we analyze the analytical models of epi- and hypocycloids described in the literature $[17,18]$.

The parametric hypocycloid equation has the same form as in (1), only with the replacement of " $+a$ " by " $-a$ " (see page 111 in [17]):

$$
\left.\begin{array}{l}
x_{M}=(A-a) \cos \varphi+a \cos \left[\frac{(A-a) \cdot \varphi}{a}\right] \\
y_{M}=(A-a) \sin \varphi-a \sin \left[\frac{(A-a) \cdot \varphi}{a}\right] .
\end{array}\right\}
$$

Here, in the second expression, the minus sign in front of $a$ is explained by the fact that the sine is an odd function [17].

Note that the derivation of the hypocycloid equations in parametric form (5) was made on page 243 in [18].

The form of the curve of a hypocycloid, similarly to an epicycloid $[17,18]$, depends on the ratio $\frac{A}{a}=m$.

It is also well known (see p. 111 in [17]) that there are elongated and shortened epi- and hypocycloids. Equation (in parametric form):

$$
\left.\begin{array}{l}
x_{M}=(A+a) \cos \varphi-\lambda a \cos \left[\frac{(A+a) \cdot \varphi}{a}\right] ; \\
y_{M}=(A+a) \sin \varphi-\lambda a \sin \left[\frac{(A+a) \cdot \varphi}{a}\right],
\end{array}\right\}
$$

where, as before, $A$ and $a$ are the radii of the fixed and moving circles, respectively; $\lambda a=$ $C M$ - the distance from the center of the moving circle $C$ to the moving point $M$ belonging to this circle (where $\lambda$ is any number, for example, for elongated $\lambda>1$, for shortened $\lambda<1$ ), and in the case of hypocycloid " $+a$ " in (6) is replaced by " $-a$ ".

\subsection{Derivation of the epicycloid equation in parametric form}

In mathematics, the derivation of the equations of the trajectory of a moving point $M$ of epiand hypocycloids is known only in [18]. The derivation of the epicycloid equation in parametric form (1) (see pages 242-144 in [18]) was made under the assumption that when the pinion cage $H$ (line $O C$ ) was rotated through angle $\varphi_{H}$, the center of rotation $C$ of the satellite (gear) 2 would be on the length of the pinion cage $H$, and the movable circle 2 rolls 
without sliding along the fixed circle 1, subject to the equality of the $\operatorname{arcs} M_{0} P$ and $P M$ (Fig. 2).

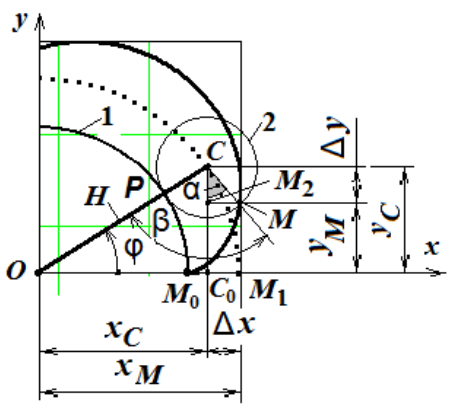

Fig. 2. To the derivation of the epicycloid equation.

In fig. 2: $M_{0}$ - the beginning of the epicycloid and the point of its return; $\varphi=\varphi_{H}$ is the angle of rotation of the pinion cage $H$, at which it will rotate along the arc $P M_{0} ; \beta$ - the smallest rotation angle (or arc coordinate) of the arc $P M$, at which the moving point $M$ has the coordinates $x_{M}$ and $y_{M} ; \alpha$ - the angle $\Delta M_{2} C M$ at the vertex $C$.

From this it is clear that the system of equations (1) is the result of purely mathematical research, and it is not possible to make any corrections to the detriment of the physical meaning of the applied problem.

\subsection{Representation of the equations of epi- and hypocycloids in parametric form as applied to the epi- and hypocyclic mechanism}

Let's rewrite system (1) and (6) as applied to the epicyclic mechanism in the form:

$$
\left.\left.\begin{array}{l}
x_{M}=(R+r) \cos \varphi_{H}-r \cos \left[\frac{(R+r) \cdot \varphi_{H}}{r}\right] ; \\
y_{M}=(R+r) \sin \varphi_{H}-r \sin \left[\frac{(R+r) \cdot \varphi_{H}}{r}\right] ;
\end{array}\right\} \begin{array}{l}
x_{M}=(R+r) \cos \varphi_{H}-\lambda r \cos \left[\frac{(R+r) \cdot \varphi_{H}}{r}\right] ; \\
y_{M}=(R+r) \sin \varphi_{H}-\lambda r \sin \left[\frac{(R+r) \cdot \varphi_{H}}{r}\right]
\end{array}\right\}
$$

Here $R$ and $r$ - the radii of a fixed and moving circle, respectively; $\varphi_{H}-$ the angle $\angle C O x$ (see Fig. 2), by which the center of the moving circle is rotated.

Let's rewrite system (1), (2) and (6) as applied to the hypocyclic mechanism in the following form:

$$
\left.\begin{array}{l}
x_{M}=(R-r) \cos \varphi_{H}+r \cos \left[\frac{(R-r) \cdot \varphi_{H}}{r}\right] ; \\
y_{M}=(R-r) \sin \varphi_{H}-r \sin \left[\frac{(R-r) \cdot \varphi_{H}}{r}\right] ;
\end{array}\right\}
$$




$$
\left.\begin{array}{l}
x_{M}=(R-r) \cos \varphi_{H}+\lambda r \cos \left[\frac{(R-r) \cdot \varphi_{H}}{r}\right] ; \\
y_{M}=(R-r) \sin \varphi_{H}-\lambda r \sin \left[\frac{(R-r) \cdot \varphi_{H}}{r}\right] .
\end{array}\right\}
$$

\subsection{Representation of the epi- and hypocycloid equation in parametric form, convenient for calculation}

As before, the shape of the curve of epi- and hypocycloids constructed according to equations (7), (8) and (9), (10) depends on the ratio $\frac{R}{r}=m$. Therefore, for the convenience of calculations, we represent the system (7) and (8), (9) and (10) as applied to the epi- and hypocyclic mechanism in the following form:

$$
\begin{aligned}
& x_{M}=(R+r) \cos \varphi_{H}-r \cos \left[(m+1) \cdot \varphi_{H}\right] ; ; \\
& \left.y_{M}=(R+r) \sin \varphi_{H}-r \sin \left[(m+1) \cdot \varphi_{H}\right] ;\right\} \\
& x_{M}=(R+r) \cos \varphi_{H}-\lambda r \cos \left[(m+1) \cdot \varphi_{H}\right] ; ; \\
& \left.y_{M}=(R+r) \sin \varphi_{H}-\lambda r \sin \left[(m+1) \cdot \varphi_{H}\right] ;\right\} \\
& \left.x_{M}=(R-r) \cos \varphi_{H}+r \cos \left[(m-1) \cdot \varphi_{H}\right] ;\right\} \\
& \left.y_{M}=(R-r) \sin \varphi_{H}-r \sin \left[(m-1) \cdot \varphi_{H}\right] ;\right\} \\
& \left.x_{M}=(R-r) \cos \varphi_{H}+\lambda r \cos \left[(m-1) \cdot \varphi_{H}\right] ;\right) \\
& y_{M}=(R-r) \sin \varphi_{H}-\lambda r \sin \left[(m-1) \cdot \varphi_{H}\right] \text {. }
\end{aligned}
$$

We emphasize that the correctness or incorrectness of the representation of equations (3), (4) and (11) - (14) in parametric form can be verified by the results of computational experiments.

Let's turn to computational experiments, which allow assessing the correctness and/or incorrectness of writing down the equations of epi- and hypocycloids in parametric form in the form of (11) - (14) along the trajectories of the movement of individual points and links of these mechanisms.

\subsection{The results of computational experiments in the Mathcad system [23]}

The initial calculation data: $R=60$ - the radius of the gear wheel 2 (or fixed circle), $\mathrm{mm} ; r$ $=20$ - radius of gear 1 (satellite and/or moving circle), $\mathrm{mm} ; \lambda=1.4$ - any number (bearing in mind that for elongated epi- and hypocycloids $\lambda>1$, and for shortened one $-\lambda<1$ ); $\omega_{H}=5$ - angular velocity of the pinion cage $H, \mathrm{rad} / \mathrm{s} ; m=R / r=3$ - is the number of branches that "go around" a fixed circle outside or inside it; $L_{H}=R+r=80$ - length of the pinion cage $H, \mathrm{~mm} ; C M=\lambda \cdot r=1.4 \cdot 20=28$ - distance from the center of the moving circle (or satellite 2) $C$ to the moving point $M$ belonging to this circle, $\mathrm{mm} ; t=t_{0}$, $\Delta t \ldots\left(2 \cdot \pi / \omega_{H}\right) n$ - the variable parameter of the travel time of pinion cage $H$ at the moment $t_{0}$ $=0 \mathrm{~s}$, where $\Delta t=0.001$ - the step of varying the travel time of the pinion cage $H, \mathrm{~s} ; n$ - the number of rotation periods of the pinion cage $H$ (any given number, for example, $n=100$ ); 
$\varphi_{H}(t)=\omega_{H} t$ - the angle of rotation of the pinion cage $H$ at time $t$ (s) relative to the axis $O x$, rad.

First, we present the projections of the characteristic points of the mechanism on the coordinate axis in the following form. fig. 2):

Projections on the coordinate axes $O x$ and $O y$ of a point $P$ lying on a fixed circle 1 (see

$$
\left.\begin{array}{l}
x R(t)=R \cos \varphi_{H}(t) \\
y R(t)=R \sin \varphi_{H}(t) .
\end{array}\right\}
$$

Projections of point $C$ lying on the trajectory of the circle of the center of satellite 2 on the coordinate axes $O x$ and $O y$ (see fig. 2):

$$
\left.\begin{array}{l}
x C(t)=(R+r) \cos \varphi_{H}(t) \\
y C(t)=(R+r) \sin \varphi_{H}(t)
\end{array}\right\}
$$

Projections of the moving point $M$ of satellite 2 of the epicycloid on the coordinate axes $O x$ and $O y$ (see equation (12), fig. 2):

$$
\left.\begin{array}{l}
x_{M}(t)=(R+r) \cos \varphi_{H}(t)-\lambda \cdot r \cos \left[(m+1) \cdot \varphi_{H}(t)\right] ; \\
y_{M}(t)=(R+r) \sin \varphi_{H}(t)-\lambda \cdot r \sin \left[(m+1) \cdot \varphi_{H}(t)\right]
\end{array}\right\}
$$

Projections of the moving point $M$ of satellite 2 of the hypocycloid on the coordinate axes $O x$ and $O y$ (see equation (14), fig. 2):

$$
\left.\begin{array}{l}
x_{M}(t)=(R-r) \cos \varphi_{H}(t)+\lambda \cdot r \cos \left[(m-1) \cdot \varphi_{H}(t)\right] ; \\
y_{M}(t)=(R-r) \sin \varphi_{H}(t)-\lambda \cdot r \sin \left[(m-1) \cdot \varphi_{H}(t)\right]
\end{array}\right\}
$$

It is well known $[22,23]$ that the law of motion of a point can be illustrated not only by curves in the coordinate system time - displacement, according to equations (15) - (18) (for an example, see Fig. 3). In some cases, it is convenient to use the phase plane to describe the motion. For the problem under consideration, the phase plane is a Cartesian coordinate system in which the displacement $\mathrm{x}_{\mathrm{M}}(\mathrm{t})$ is plotted along the abscissa axis and $\mathrm{y}_{\mathrm{M}}(\mathrm{t})$ - along the ordinate axis. In this plane, a phase trajectory can be obtained, i.e. the geometric location of the image points (for example, points $O, P, C$, and $M$ ) corresponding to consecutive moments of time $t$.

Below we present the results of constructing the phase trajectories of the image points in the Mathcad system [23].

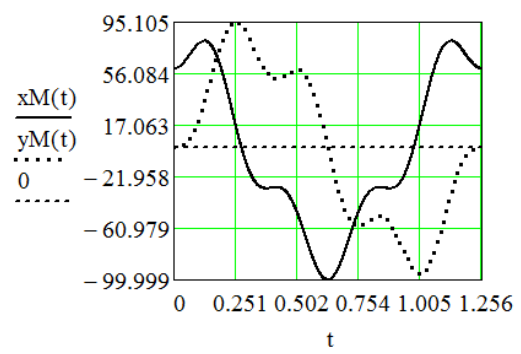

Fig. 3. The law of motion of the point $M$ in the coordinate system time - displacement at $\mathrm{m}=3$ and $\lambda$ $=1.0$. 
For given initial data of the problem, the graphical representations of equations (15) (18) for $m=3$ and $\lambda=1.0$ have the form shown in fig. 4-6.

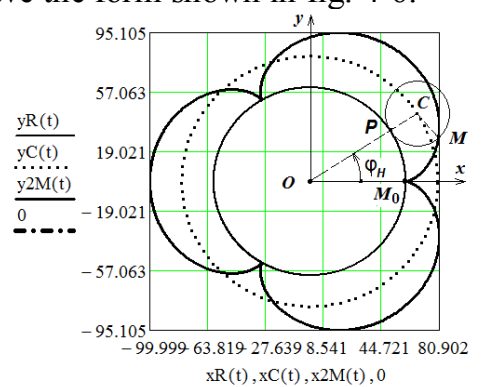

Fig. 4. Phase trajectories of the image points $\mathrm{P}, \mathrm{C}$, and $\mathrm{M}$ for $\mathrm{m}=3$ and $\lambda=1.0$.

In fig. 4 , phase trajectories of the image points $P\left(\mathrm{M}_{0}\right.$ is the return point) and $C$, as follows from equations (15) and (16), correspond to circles with center $O$, and the trajectory of the image point $M$, according to equation (16), corresponds to an epicycloid with three branches, since $m=3$ and $\lambda=1.0$ (see fig. 54, $a$ in [17]).

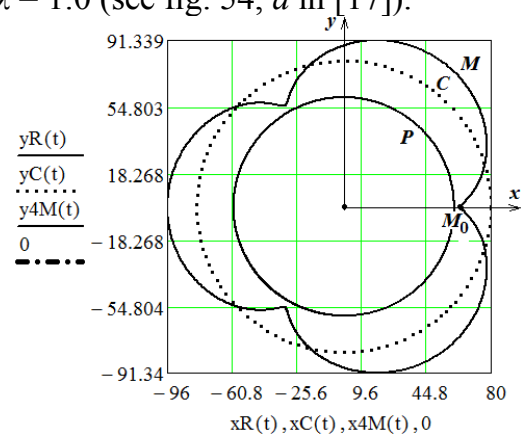

Fig. 5. Phase trajectories of the image points $\mathrm{P}, \mathrm{C}$, and $\mathrm{M}$ for $\mathrm{m}=3$ and $\lambda=0.8$.

It is obvious that in fig. 5, the phase trajectory of the image point $M$, according to equation (18), corresponds to a shortened epicycloid with three branches, since $m=3$ and $\lambda$ $=0.8$ (see fig. 56, $c$ in [17]).

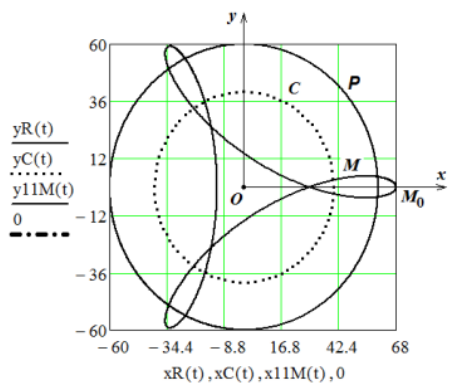

Fig. 6. Phase trajectories of the image points $P, C$, and $M$ for $m=3$ and $\lambda=1.4$.

As can be seen, the phase trajectory of the image point $M$ shown in fig. 6 , according to equation (18), corresponds to an elongated hypocycloid with three branches, since $m=3$ and $\lambda=1.4$ (see fig. 55, $\mathbf{a}$ in [17]).

The phase trajectories of the image points $P, C$, and $M$ (see fig. 4) for $m=3$ and $\lambda=1.0$, as well as for $u_{21}=-m$, constructed according to the system of equation (3) [14, 22], are presented in fig. 7. 


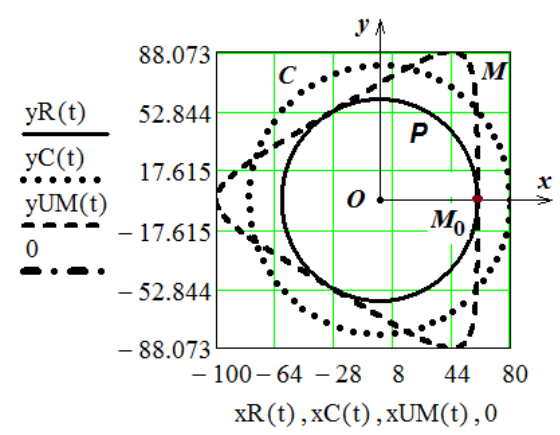

Fig. 7. Phase trajectories of the image points $P, C$, and $M$ for $u 21=m=-3$ and $\lambda=1.0$.

As can be seen, the phase trajectories of the image points $P, C$, and $M$ shown in Fig. 4 and 7 are fundamentally different from each other, which confirms the incorrectness of equations of type (3) in $[14,22]$ and the inadmissibility of making any corrections to the classical equations of epi- and hypocycloids in parametric form. For this reason, all the results of studies to determine the kinematic characteristics of individual points of the connecting rod 3 and slider 4 of the two-wire Assur group (see fig. 1) performed in [12] are scientifically untenable. In addition, all the mathematical calculations in [20] on determining the speed and acceleration of the moving point of the satellite of both the planetary and differential mechanisms (see pages 274-276) are also incorrect.

\section{Conclusions}

Thus, based on the conducted studies, we especially note the following results:

1. the equations of epi- and hypocycloids in parametric form are presented with reference to the epi- and hypocyclic mechanism in a form convenient for calculation.

2 . the results of computational experiments on constructing phase trajectories of a moving point $M$ of epi- and hypocycloids with analysis of the obtained curves are presented. Moreover, the inadmissibility of making any adjustments to the classical epicycloid equations in parametric form is proved.

3. summarizing the analysis of phase trajectories depicting the characteristic points $P, C$ and $M$ of the epi- and hypocycloids shown in fig. 3-6, it was revealed that they are identical with similar trajectories given in [15], which confirm the correctness and fundamental nature of the mathematical records of equations (15) - (18).

The analytical model of epi- and hypocycloids is of practical importance, since it allows designing geared linkage mechanisms formed by connecting the planetary mechanism, as the primary mechanism, with two-wire Assur groups of various modifications (for example, such as RRT, RRR, RTR) [15, 16].

\section{References}

1. L.G. Loytsynsky, L.I. Lurye, Theoretical machines (The state technical-theoretical publishing house, Moscow, 1932)

2. I.V. Meshchersky, Problems of the Theoretical mechanics (Lany, St. Peterburg, 1998)

3. V.P. Agrawal, J.S. Rao, Mech. Mach. Theory 22, 497-504 (1987)

4. J. Chu, W. Cao, Mech. Mach. Theory 29, 53-58 (1994)

5. M. Kolovsky, A. Evgrafov, Yu. Semenov, A. Slousch, Advanced Theory of Mechanisms and Machines (Springer, Berlin, 2000) 
6. J.J. Uicker, G.R. Pennock, Theory of Mechanisms (Oxford Univ. Press, New York, 2003)

7. Kh.T. Turanov, V.N. Anferov, Sh.Kh. Turanov, V.U. Ignatugin, Theory of mechanisms and machines 2(3), 78-84 (2005)

8. A.B.S. Rao, A. Srinath, A.C. Rao, Institution of Engineers 86, 195-201 (2006)

9. Kh.T. Turanov, V.N. Anferov, Sh.Kh. Turanov, V.U. Ignatugin, Theory of mechanisms and machines 2(5), 85-92 (2007)

10. S.S.H. Rizvi, A. Hasan, R.A. Khan, Int. J. Mechanisms and Robotic Systems, Inderscience Enterprises Ltd 3(1), 48-59 (2016)

11. V.I. Pozhbelko, E. Kuts, Theory of mechanisms and machines 4(16), 139-149 (2018)

12. V.I. Pozhbelko, E. Kuts, Mechanism and Machine Science 57, 27-35 (2018)

13. A. Hasan, International Journal of Computational Engineering Research 1(8), 13-19 (2018)

14. R.I. Karimov, N.N. Begimov, Simulation of kinematics and kinetostatical cyclic mechanisms (Tashkent State Polytechnic University, Tashkent, 2015)

15. K.B. Levenson, Theory mechanisms and machines (Mashgiz, Leningrad, 1954)

16. I.I. Artobolevsky, Theory mechanisms and machines (Science, Moscow, 1975)

17. I.N. Bronshteyn, K.A. Semendyev, Handbook of mathematics for engineers and students of higher education: Textbook the village (Lany, St. Petersburg, 2009)

18. N.N. Luzin, Differential calculus (Soviet science, Moscow, 1958)

19. I.M. Vinogradov, Mathematical encyclopedia (Soviet encyclopedia, Moscow, 1984)

20. Yu.V. Prokhorov, Mathematical encyclopedia (Soviet encyclopedia, Moscow, 1988)

21. M.V. Sablikov, Cotton harvesters (Agropromizdat, Moscow, 1985)

22. Kh.Kh. Usmankhodjaev, Theory mechanisms and machines. Textbook of higher education (Teacher, Tashkent, 1981)

23. E.G. Makarov, Mathcad: Training course (+CD) (Piter, St. Petersburg, 2009)

24. Ya.G. Panovko, Fundamentals of applied oscillations and shock (Polytechnics, Leningrad, 1990)

25. G.A. Bugaenko, V.V. Malanin, V.I. Yakovlev, Fundamentals of classical mechanics (Higher school, Moscow, 1999) 